\title{
Multidetector computed tomography-derived emphysema index for preoperative quantification of emphysema severity
}

\author{
Shvetank Agarwal, MD (1) A Amjad Najim, MD • Jayanth H. Keshavamurthy, MD
}

Received: 10 June 2016/Revised: 13 June 2016/Accepted: 20 June 2016/Published online: 28 June 2016

(c) Canadian Anesthesiologists' Society 2016

Emphysema is a chronic obstructive airway disease (COPD) characterized by loss of elastic recoil of the lungs, which results in alveolar hyperinflation and collapse of the airways during exhalation. This disease presents multiple challenges during anesthesia, and assessing its severity preoperatively may help with intraoperative management.

Pulmonary function tests provide only functional information, and conventional chest radiography provides only a gross estimation of disease severity. Multidetector computed tomography (MDCT) combined with dedicated post-processing software however, is a novel tool that utilizes high-resolution imaging, thereby allowing quantitative assessment of emphysema. This rapidly evolving technology provides detailed anatomical information about lung parenchyma, the airways, and the distribution of emphysematous lung tissue. ${ }^{1}$ It requires no intravenous contrast material and can be used to differentiate between airway-predominant and emphysema-predominant COPD. ${ }^{2}$

Quantitatively, it allows assessment of the density of each voxel (three-dimensional [3D] volume unit representing a pixel on the 2D image) of lung parenchyma using vendor-specific software that calculates total lung volume (TLV) and emphysematous volume (EV)

S. Agarwal, MD $(\bowtie) \cdot$ A. Najim, MD

Department of Anesthesiology and Perioperative Medicine,

Medical College of Georgia, Augusta University, Augusta, GA,

USA

e-mail:sagarwal@augusta.edu

J. H. Keshavamurthy, MD

Department of Radiology, Medical College of Georgia, Augusta University, Augusta, GA, USA of both lungs. ${ }^{3}$ The typical value for the attenuation coefficient, Housefield units, is set at -950 to differentiate between emphysema (seen as red dots) and normal lung tissue (Figure). ${ }^{3}$ The EV/TLV or the emphysema ratio (ER) (\%) can then be calculated for individual lobes of the lungs. ${ }^{2}$ The Figure shows an overall ER of $17.1 \%$, which signifies that at least $17 \%$ of the patient's lungs are not

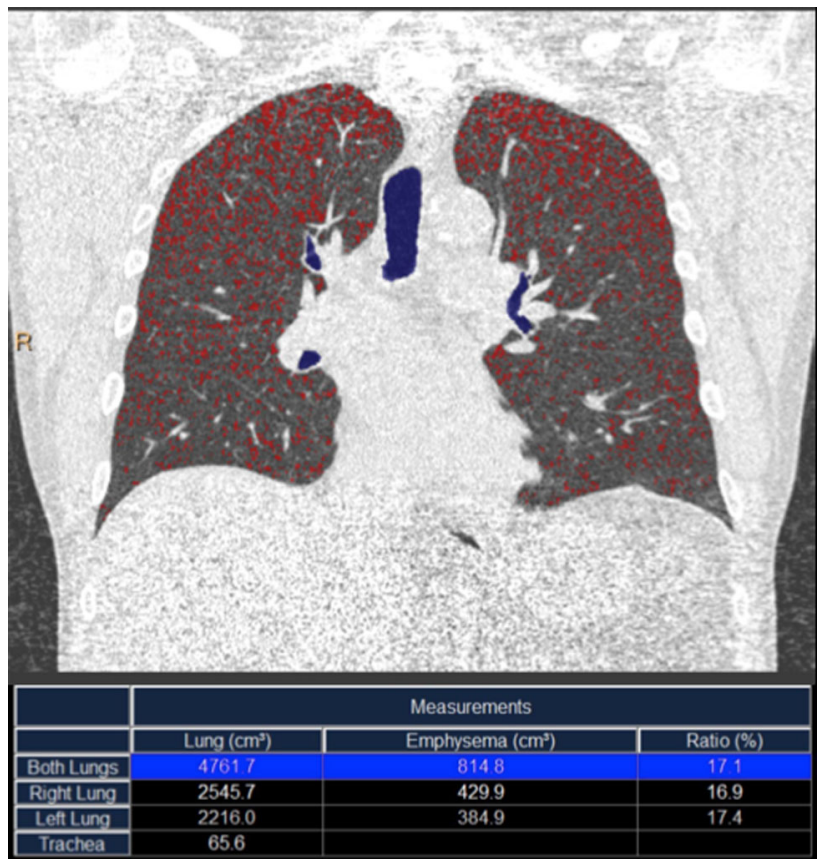

Figure Multidetector non-contrast coronal reformatted chest computed tomography image shows areas of air-trapping in red. The table in the lower aspect of the image reflects the ratio between the air-trapped emphysematous volume (EV) and normal lung volumes. The blue areas are the large airways and were not included in the calculation of this patient's $17.1 \% \mathrm{EV}$ ratio. 
available for active air exchange while at the same time being more susceptible to developing pneumothorax due to air-trapping. There is considerable evidence to support intraoperative use of protective ventilatory strategies, including lower tidal volumes $\left(6-7 \mathrm{~mL} \cdot \mathrm{kg}^{-1}\right.$ for two-lung ventilation and 3-4 $\mathrm{mL} \cdot \mathrm{kg}^{-1}$ for one-lung ventilation) to reduce volutrauma in general. ${ }^{4}$ In the patient represented in the Figure, however, we attempted to further reduce tidal volumes by approximately $17 \%$ to less than $6 \mathrm{~mL} \cdot \mathrm{kg}^{-1}$ for two-lung ventilation and less than $3 \mathrm{~mL} \cdot \mathrm{kg}^{-1}$ for one-lung ventilation.

There are multiple limitations to using MDCT, including the lack of availability of the software in smaller community hospitals as well as the lack of standardization among software developers. Also, although it takes less than a minute for an experienced radiologist to calculate the volumes, not every patient has undergone preoperative non-contrast $\mathrm{CT}$ of the chest, and the ER is not routinely reported by radiologists unless specifically requested, reducing its timely availability for the anesthesiologist's use in many cases. Nevertheless, there is a potential for this novel technology to help anesthesiologists and intensivists selecting the appropriate tidal volume, thereby avoiding volutrauma. We urge the anesthesia community to design and carry out research studies to validate this technology.

Conflicts of interest None declared.

Editorial responsibility This submission was handled by Dr. Hilary P. Grocott, Editor-in-Chief, Canadian Journal of Anesthesia.

\section{References}

1. Matsuura $Y$, Kawata $N$, Yanagawa $N$, et al. Quantitative assessment of cross-sectional area of small pulmonary vessels in patients with COPD using inspiratory and expiratory MDCT. Eur J Radiol 2013; 82: 1804-10.

2. Pauls S, Gulkin D, Feuerlein S, et al. Assessment of COPD severity by computed tomography: correlation with lung functional testing. Clin Imaging 2010; 34: 172-8.

3. Karia $S$, Mahadeva $R$, Balan A, Babar J. The evolving role of MDCT in the assessment of patients with chronic obstructive pulmonary disease. Clin Radiol 2015; 70: 752-9.

4. Kilpatrick $B$, Slinger $P$. Lung protective strategies in anaesthesia. Br J Anaesth 2010; 105(Suppl 1): i108-16. 